Fetal Diagnosis and Therapy
Fetal Diagn Ther 2018;44:299-304

DOI: $10.1159 / 000484317$
Received: August 15, 2017

Accepted after revision: October 17, 2017

Published online: November 8, 2017

\title{
Prenatal Screening for 22q11.2 Deletion Using a Targeted Microarray-Based Cell-Free DNA Test
}

\author{
Maximilian Schmid ${ }^{a}$ Eric Wang ${ }^{a}$ Patrick E. Bogard ${ }^{a}$ Elisa Bevilacquab \\ Coleen Hacker ${ }^{a}$ Susie Wang ${ }^{a}$ Jigna Doshi ${ }^{a}$ Karen White ${ }^{a}$ Jennifer Kaplan ${ }^{a}$ \\ Andrew Sparks ${ }^{a}$ Jacques C. Jani ${ }^{b}$ Renee Stokowski ${ }^{a}$ \\ ${ }^{a}$ Ariosa Diagnostics Inc., Roche Sequencing Solutions, Inc., San Jose, CA, USA; b Department of Obstetrics and \\ Gynecology, University Hospital Brugmann, Université Libre de Bruxelles, Brussels, Belgium
}

\section{Keywords}

22q11.2 deletion · NIPT · Cell-free DNA · Microdeletion

\begin{abstract}
Objective: To determine the performance of a targeted microarray-based cell-free DNA (cfDNA) test (Harmony Prenatal Test ${ }^{\circledR}$ ) for the identification of pregnancies at increased risk for 22q11.2 deletion. Methods: Test performance was determined in 2 steps including a total of 1,953 plasma samples. Analytical validation was performed in 1,736 plasma samples. Clinical verification of performance was performed in an additional 217 prospectively ascertained samples from pregnancies with fetal deletion status determined by diagnostic testing. Results: Analytical sensitivity was 75.4\% (95\% Cl: 67.1-82.2\%) based on 122 samples with deletions ranging from 1.96 to $3.25 \mathrm{Mb}$. In 1,614 presumed unaffected samples, specificity was determined to be at least $99.5 \%(95 \% \mathrm{Cl}$ : 99.0-99.7\%). In the clinical cohort, 5 of 7 samples from pregnancies affected with 22q11.2 deletion were determined to have a high probability of deletion. There were no false positive results in the 210 unaffected samples in this cohort. These clinical data are consistent with the performance demonstrated in the analytical validation. Conclusions:
\end{abstract}

cfDNA testing using a targeted microarray-based technology is able to identify pregnancies at increased risk for $22 q 11.2$ deletions of $3.0 \mathrm{Mb}$ and smaller while maintaining a low false positive rate.

(c) 2017 The Author(s)

Published by S. Karger AG, Basel

\section{Introduction}

The analysis of cell-free DNA (cfDNA) in maternal plasma is an effective method for trisomy 21 screening in the general obstetrical population [1]. It has also demonstrated high sensitivity and specificity in the detection of rarer fetal autosomal trisomies such as trisomy 18 and 13 $[2,3]$. Trisomy screening using cfDNA analysis is consequently being integrated as a routine option in prenatal care $[4,5]$. This technology has advanced rapidly and researchers are seeking to capitalize on the power of cfDNA analysis to screen for a broader range of conditions in pregnancy. Just as diagnostic genetic testing has evolved with the diagnosis of genetic conditions that have etiologies of an increasingly smaller scale (from microscopic to submicroscopic to molecular), investigators have explored expanding cfDNA testing in the same direction. In

\begin{tabular}{ll}
\hline KARGER & $\begin{array}{l}\text { (c) 2017 The Author(s) } \\
\text { Published by S. Karger AG, Basel }\end{array}$ \\
E-Mail karger@karger.com & This article is licensed under the Creative Commons Attribution- \\
www.karger.com/fdt & $\begin{array}{l}\text { NonCommercial-NoDerivatives 4.0 International License (CC BY- } \\
\text { NC-ND) (http://www.karger.com/Services/OpenAccessLicense). } \\
\text { Usage and distribution for commercial purposes as well as any dis- } \\
\text { tribution of modified material requires written permission. }\end{array}$
\end{tabular}

Maximilian Schmid, MD

Roche Sequencing Solutions

5945 Optical Court, San Jose, CA 95138 (USA)

E-Mail maximilian.schmid@ roche.com 
addition to whole-chromosome aneuploidy, there is interest in screening for conditions caused by submicroscopic chromosome changes, such as microdeletions, and single-gene disorders. However, with the use of cfDNA as a screening test, there needs to be careful consideration in the implementation of cfDNA testing: target diseases need to be common and of sufficient clinical relevance; and a test should have sufficiently low false positive rates to maximize the positive predictive value (PPV) of the test and keep invasive testing rates low.

Most microdeletions are relatively rare, with prevalence usually ranging from 1 in 10,000 to 1 in 50,000 [6]. The most common microdeletion syndrome is the $22 \mathrm{q} 11.2$ deletion syndrome, a multisystem disorder caused by a submicroscopic deletion on the long arm of chromosome 22. Common phenotypic findings include growth and developmental delay, cardiac defects, cleft palate, recognizable facial features, learning disabilities, and immunodeficiency $[7,8]$. 22q11.2 deletion has a variable clinical presentation and has been identified as the common underlying etiology of conditions previously described as $\mathrm{Di}$ George syndrome and velocardiofacial syndrome (VCFS), among others [9]. Estimates of the prevalence for 22q11.2 deletion range from approximately 1 in 4,000 to 1 in 10,000 live births [10]; however, recent publications of prenatal series have reported a prevalence as high as 1 in $1,000[11,12]$. Overall it is the second most common genetic cause of developmental delay and major congenital heart disease after Down syndrome [13] and is more common than trisomy 18 and trisomy 13 combined. Most affected individuals (85\%) have a deletion of $3 \mathrm{Mb}$ that encompasses approximately 45 functional genes. A smaller subset of patients present with "atypical" or "nested" deletions that are usually within the $3 \mathrm{Mb}$ region [8].

To date, commonly used prenatal screening and testing methods generally do not reliably identify pregnancies at increased risk for 22q11.2 deletion syndrome. Of note, maternal age is not a risk factor, unlike fetal trisomy. Family history is also not a good predictor of risk as more than $90 \%$ of individuals with $22 \mathrm{q} 11.2$ deletion have a de novo mutation [10]. Moreover, the deletion is not detected by serum screening. Although routine ultrasound examination may identify associated findings such as cardiac defects, the sensitivity is difficult to estimate and diagnosis may be delayed until late in gestation. Invasive diagnostic procedures will only detect $22 \mathrm{q} 11.2$ deletions if fluorescent in situ hybridization (FISH) or whole-genome microarray technology is used. Prenatal screening for 22q11.2 deletions using cfDNA would enable the early identification of pregnancies at increased risk for the deletion syndrome. Early diagnosis may inform prenatal management as well as lead to earlier intervention and potentially improved postnatal outcomes.

The Harmony Prenatal Test ${ }^{\circledR}$ screens for fetal aneuploidy by using a directed technology (DANSR ${ }^{\mathrm{TM}}$ ) to target specific regions of chromosomes $21,18,13, \mathrm{X}$, and Y and quantify them on a custom microarray $[14,15]$. A fetal fraction responsive algorithm (FORTE ${ }^{\mathrm{TM}}$ ) incorporates this information, the relative proportion of fetal cfDNA, and maternal-specific factors to calculate probability scores for trisomy 21 , trisomy 18 , trisomy 13 , and the sex chromosome aneuploidies [16].

The objective of this study was to evaluate the ability of DANSR and FORTE to identify fetal 22q11.2 deletions after adding DANSR assays within a $3.0 \mathrm{Mb}$ region of chromosome 22q11.2. Analytical sensitivity was investigated in simulated pregnancy samples and maternal plasma from pregnancies with confirmed fetal 22q11.2 deletions. Minimum test specificity was established in a large group of samples from presumed unaffected pregnancies. The assay was further verified in an additional set of prospectively ascertained samples from pregnancies with fetal deletion status determined by diagnostic testing.

\section{Materials and Methods}

Samples for Determination of Analytical Performance

All patients provided consent under clinical study protocols AD-001, AD-202, and TD-006. All protocols were reviewed and approved by the appropriate institutional review board or ethics committee and were conducted per International Council for Harmonisation and Good Clinical Practice guidelines.

1,736 plasma samples were used to determine the ability of DANSR and FORTE to assess for 22q11.2 deletions. 122 samples had confirmed deletions; 1,614 samples were maternal plasma from pregnancies with no known diagnosis of fetal or maternal $22 \mathrm{q} 11.2$ deletion.

Of the 122 plasma samples with confirmed deletions, 120 were simulated pregnancy samples and 2 were prospectively collected samples from pregnant women. All deletions were determined based on chromosomal microarray analysis using the Agilent CGX v1.1 v1.1 8-plex array, Agilent CGH ISCA 60K array, or the Affymetrix CytoScan $750 \mathrm{~K}$ array. The 22q11.2 deletion sizes ranged from 1.96 to $3.25 \mathrm{Mb}$.

The simulated pregnancy plasma samples were generated by combining plasma from an adult with a confirmed 22q11.2 deletion with plasma from a related or unrelated nonpregnant female adult with no diagnosis of a 22q11.2 deletion. The plasma samples used were from a total of 14 affected individuals, 2 males and 12 females, and 44 unaffected females, of which 9 are the natural mothers of the affected subjects. Plasma from each affected individual was mixed with individual plasma aliquots from up to 4 different nonpregnant female plasma sources to achieve simulated fetal fractions from 4 to $33 \%$. The fetal fractions ranged across a 
Fig. 1. 22q11.2 chromosomal region and

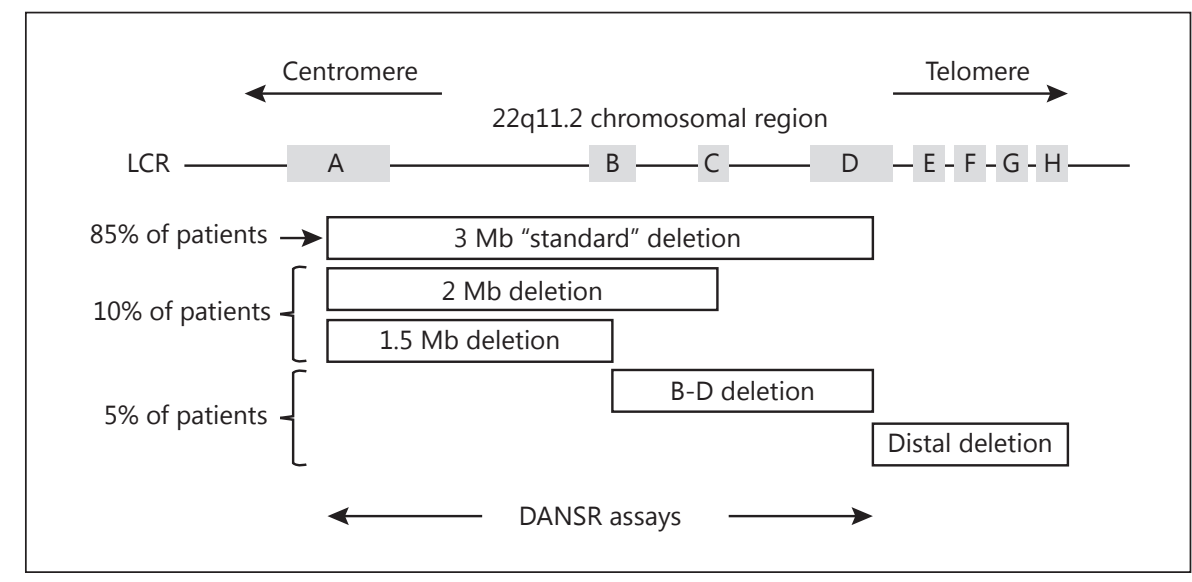
location of DANSR assays.

natural distribution with approximately $30 \%$ of the samples less than $10 \%, 40 \%$ between 10 and $15 \%$, and $30 \%$ greater than $15 \%$ fetal fraction.

A series of 1,614 prospectively ascertained maternal plasma samples with no known diagnosis of fetal or maternal 22q11.2 deletions were used to evaluate analytical specificity. These samples were submitted to the Ariosa Diagnostics CLIA laboratory for clinical cfDNA testing for fetal trisomy using the Harmony Prenatal Test. All were singleton pregnancies, unselected for clinical indication or in vitro fertilization status, which received a Harmony result. Samples were de-identified and anonymized before study. Subjects consented to the use of their specimens for additional studies, including test validation.

\section{Samples for Clinical Verification}

Plasma samples from women carrying pregnancies with known fetal 22q11.2 deletion status were obtained from the sample bank created as part of the RAPID project (RP-PG-0707-10107) with national research ethics approval (13/LO/0082). All participants gave written consent. Blood samples were collected prospectively into either Streck or EDTA tubes, double spun, and plasma stored at $-80^{\circ} \mathrm{C}$ [17]. Seven samples from pregnancies with $22 \mathrm{q} 11.2$ deletions and 210 samples from confirmed unaffected pregnancies were included in the analysis. 22q11.2 deletions were diagnosed by either FISH (4 samples) or chromosomal microarray (3 samples) with none identified as atypical or nested deletions. All unaffected pregnancies had normal chromosomal microarray analysis.

\section{Design and Microarray Quantification of DANSR Assay}

Products

Samples were processed as previously described for the Harmony Prenatal Test [14-16]. Approximately 500 additional DANSR assays were designed against targets uniformly distributed within a $3.0 \mathrm{Mb}$ region of 22q11.2 between low copy repeats (LCR) A and D (Fig. 1). Custom DNA microarrays were manufactured by Affymetrix Inc. (Santa Clara, CA, USA) to specifically quantify all DANSR assay products. Each sample was analyzed on a single custom microarray.

\section{Data Analysis}

The previously published FORTE algorithm was modified to calculate the probability of a deletion within the $22 \mathrm{q} 11.2$ region similar to that previously described for chromosomes 21,18 , and 13 where the algorithm generates a probability score that incorporates fetal fraction [16]; however, unlike with trisomy assessment, there is no adjustment based on maternal or gestational age. The algorithm was not modified to calculate the probability of a duplication in this region.

The probability of a 22q11.2 deletion was determined blinded to ground truths in 122 samples with confirmed 22q11.2 deletions and 1,614 presumed unaffected samples. After optimization of the algorithm using the analytical validation dataset, the probability of a deletion was assessed blinded in the clinical verification cohort, which included a total of 226 plasma samples from pregnancies with known 22q11.2 deletion status. Samples with probability scores exceeding 1\% were classified as "high probability" for a 22 q11.2 deletion, which can be fetal or maternal, or both. Otherwise, the sample was classified as "no evidence of a deletion observed." All confidence intervals were determined using the Wilson method.

\section{Results}

Results are shown in Table 1.

\section{Analytical Validation}

Analytical sensitivity was determined in 122 samples with confirmed 22q11.2 deletions. 92 of the 122 were determined to have a high probability of a deletion. This corresponds to a sensitivity of $75.4 \%$ (95\% CI: $67.1-$ $82.2 \%)$ in samples with confirmed deletions. The smallest deletion size detected was $1.96 \mathrm{Mb}$, and deletions were detected across the entire range of fetal fractions. There was no correlation between sensitivity and deletion size.

In the 1,614 samples from presumed unaffected pregnancies, the maternal age ranged from 18 to 52 years with a median of 33 years; gestational age ranged from 10 to 40 weeks with a median of 13 weeks. 1,606 were determined to have no evidence of a deletion. A greater than $1 \%$ prob- 
Table 1. Sensitivity and specificity of 22q11.2 deletion analysis using targeted microarray cfDNA testing

\begin{tabular}{lccc}
\hline & Analytical validation & Clinical verification & Combined \\
\hline Total samples, $N$ & 1,736 & 217 & 1,953 \\
22q11.2, $n / N$ & $92 / 122$ & $5 / 7$ & $97 / 129$ \\
No evidence of a deletion, $n / N$ & $1,606 / 1,614^{\text {a }}$ & $210 / 210$ & $1,816 / 1,824$ \\
Sensitivity, \% (95\% CI) & $75.4(67.1-82.2)$ & $71.4(35.9-91.8)$ & $75.2(67.1-81.8)$ \\
Specificity, \% (95\% CI) & $99.5(99.0-99.7)$ & $100(98.2-100)$ & $99.6(99.1-99.8)$ \\
\hline \multicolumn{2}{c}{ a Presumed normals - commercial samples without a known diagnosis of 22q11.2 deletion, presumed to be } \\
unaffected (may cause underestimation of specificity).
\end{tabular}

ability for a deletion was reported in 8 samples. This corresponds to a specificity of at least $99.5 \%$ (95\% CI: 99.0 $99.7 \%)$. The specificity must be considered a lower-bound estimate because these were clinical samples without the confirmed absence of a maternal or fetal deletion. The clinical samples were anonymized and unselected for clinical indication; therefore, the group of 8 samples classified as "false positives" may include true positive samples from undiagnosed mothers or pregnancies affected with a fetal 22q11.2 deletion.

\section{Clinical Verification}

In 217 samples from pregnancies with known 22q11.2 deletion status as determined by FISH or microarray, 5 of the 7 samples with deletions were identified as having a high probability of a deletion. No evidence of a deletion was observed in all 210 unaffected samples. Harmony performance in this clinical cohort is consistent with the performance observed in the analytical validation set.

\section{Discussion}

Screening for subchromosomal abnormalities by cfDNA testing has been challenged by the ability to distinguish a copy number change in a relatively small portion of the genome. Methodologies using massively parallel sequencing are impacted by read depth and size of the fetal copy number variant $[18,19]$. Sequencing depth becomes a particular issue for test performance when testing for deletions of relatively small size such as the 22q11.2 deletion. Read depth has also been observed to impact specificity with the use of single nucleotide polymorphism-based screening for 22q11.2 deletions [20]. The targeted approach to cfDNA testing exemplified by DANSR technology offers advantages over whole-ge- nome approaches because additional targets can be added and concentrated to interrogate genomic regions of specific interest such as 22q11.2.

In the present study we have expanded the Harmony Prenatal Test by adding DANSR assay targets within a 3.0 $\mathrm{Mb}$ region of 22q11.2 and investigated its ability to identify deletions within this region. Test performance was determined in 2 steps including a total of 1,953 plasma samples.

cfDNA testing for 22q11.2 deletion syndrome is currently commercially available but with only limited published prospective validation data [21]. The design of the current study is in contrast to reports of clinical laboratory experience with retrospective follow-up of samples tested [22, 23]. Retrospective studies cannot address test sensitivity because they are limited by incomplete followup and ascertainment bias. For example, a preponderance of samples with a maternal component was identified as a potential source of bias in one such study [23].

Deletion size is an important consideration when evaluating test sensitivity. Deletions in the 22q11.2 region are the result of nonallelic homologous recombination between large blocks of LCR. The most common deletion is $3 \mathrm{Mb}$ and occurs between the 2 largest repeats, LCR22A and LCR22D. Approximately $85 \%$ of affected individuals have this deletion. Smaller subsets of patients have smaller nested deletions that occur between other LCR within the same region (Fig. 1) [24]. The region covered by the DANSR assays includes both the common and nested deletions. Deletions that do not overlap with this region have been reported but are rare. Performance of a cfDNA screen for 22q11.2 deletion will be limited by the fetal deletion size that it can detect. In other words, a test that cannot detect deletions smaller than $3 \mathrm{Mb}$ will by definition have a detection rate of less than $85 \%$ [6]. To reflect clinical reality this study included samples with the typi- 
cal $3 \mathrm{Mb}$ deletion as well as samples with smaller nested deletions, and test sensitivity must be seen in this context. In fact, one could argue that sensitivity reported for comparable cfDNA tests should be adjusted if established only based on the common $3 \mathrm{Mb} 22 \mathrm{q} 11.2$ deletion.

One limitation of our dataset is that assessment of sensitivity predominately relies on simulated 22q11.2 deletion samples and specificity on presumed normal samples with no known diagnosis of fetal or maternal 22q11.2 deletion. Obtaining sufficient maternal plasma samples from affected pregnancies to determine sensitivity is a significant challenge when validating a cfDNA test for $22 \mathrm{q} 11.2$ deletion. There are only limited numbers of pregnancies ascertained prenatally that can be used for clinical validation studies. In this study, we have therefore augmented affected pregnancy samples with simulated pregnancy samples. The use of contrived samples has been reported previously; however, these studies used artificially sheared genomic DNA (enzymatically or by sonication) derived from only 1 or 2 individuals with $22 \mathrm{q} 11.2$ deletions [21, 25]. By mixing cfDNA containing plasma from affected individuals with plasma of nonpregnant women, the simulated pregnancy samples in this study represent plasma with naturally occurring cfDNA fragments. Although this may not replicate the exact fragmentation patterns observed with fetal cfDNA, we believe this approach reflects the true biologic state in pregnancy more accurately than previously reported techniques.

Likewise, obtaining pregnancy samples with the confirmed absence of a deletion in order to assess specificity is also a challenge for test validation because microarray or FISH for the 22q11.2 deletion is not standard of care for routine prenatal diagnosis. Specificity is a critical performance metric in the demonstration of test utility. Both disease prevalence and specificity contribute to the PPV, a clinically relevant statistical measure that indicates how likely pregnancies that test positive are to have a fetus affected by a condition. Although the PPV of the test will need to be demonstrated in clinical studies, the higher the specificity, the higher the PPV of a screening test will be. To achieve sufficient power to provide a reliable measurement of test specificity, test validation should include as many unaffected pregnancies as possible. To achieve this, the analytical validation set included over 1,600 plasma samples from presumed unaffected pregnancies. The outcome is a specificity of $99.5 \%$ with a tight confidence interval. It is a conservative estimate of specificity because the presumed unaffected samples come from a clinical screening population. With a pregnancy population prevalence of 1 in 1,000 and the possibility of high-risk pregnancies tested, one would expect one or more true affected pregnancies in the cohort. Thus, the true specificity is therefore likely to be higher than $99.5 \%$.

In summary, we have determined the analytical performance of a targeted microarray-based cfDNA test for assessment of fetal 22q11.2 deletions of $3 \mathrm{Mb}$ and smaller and have confirmed this performance in a cohort of prospectively collected pregnancy samples with known deletion status. Pregnancies at increased risk for 22q11.2 deletions can be identified while maintaining a high specificity. Demonstration of specificity for any potential cfDNA test expansion is critical when implementing screening in low-risk populations. Large-scale prospective clinical validation studies remain to be performed as it is possible that true clinical performance in routine screening populations may be higher.

\section{Acknowledgements}

We thank Lyn Chitty, MD, Great Ormond Street Hospital for Children NHS Foundation Trust, London, for supplying the samples used in the clinical verification cohort. We are also especially grateful to the individuals and families providing blood samples for the analytical validation portion of the study.

\section{Disclosure Statement}

M.S., E.W., P.E.B., C.H., S.W., J.D., K.W., J.K., A.S., and R.S. are employees of Roche Sequencing Solutions, Inc.

\section{Funding Sources}

This work was funded by Roche Sequencing Solutions, Inc.

\section{References}

1 Norton ME, Jacobsson B, Swamy GK, Laurent LC, Ranzini AC, Brar H, Tomlinson MW, Pereira L, Spitz JL, Hollemon D, Cuckle H, Musci TJ, Wapner RJ: Cell-free DNA analysis for noninvasive examination of trisomy. $\mathrm{N}$ Engl J Med 2015;372:1589-1597.

2 Gil MM, Quezada MS, Revello R, Akolekar R, Nicolaides KH: Analysis of cell-free DNA in maternal blood in screening for fetal aneuploidies: updated meta-analysis. Ultrasound Obstet Gynecol 2015;45:249-266.

3 Stokowski R, Wang E, White K, Batey A, Jacobsson B, Brar H, Balanarasimha M, Hollemon D, Sparks A, Nicolaides K, Musci TJ: Clinical performance of non-invasive prenatal testing (NIPT) using targeted cell-free DNA analysis in maternal plasma with microarrays or next generation sequencing (NGS) is consistent across multiple controlled clinical studies. Prenat Diagn 2015;35:1243-1246. 
4 ACOG Committee on Genetics: Committee Opinion No. 640: Cell-free DNA screening for fetal aneuploidy. Obstet Gynecol 2015; 163:e31-e37.

5 Gregg AR, Skotko BG, Benkendorf JL, Monaghan KG, Bajaj K, Best RG, Klugman S, Watson MS: Noninvasive prenatal screening for fetal aneuploidy, 2016 update: a position statement of the American College of Medical Genetics and Genomics. Genet Med 2016;18: 1056-1065.

6 Yaron Y, Jani J, Schmid M, Oepkes D: Current status of testing for microdeletion syndromes and rare autosomal trisomies using cell-free DNA technology. Obstet Gynecol 2015;126: 1095-1099.

7 McDonald-McGinn DM, Kirschner R, Goldmuntz E, Sullivan K, Eicher P, Gerdes M, Moss E, Solot C, Wang P, Jacobs I, Handler S, Knightly C, Heher K, Wilson M, Ming JE, Grace K, Driscoll D, Pasquariello P, Randall P, Larossa D, Emanuel BS, Zackai EH: The Philadelphia story: the 22q11.2 deletion: report on 250 patients. Genet Couns 1999;10: $11-24$.

8 Bassett AS, McDonald-McGinn DM, Devriendt K, Digilio MC, Goldenberg P, Habel A, Marino B, Oskarsdottir S, Philip N, Sullivan $\mathrm{K}$, Swillen A, Vorstman J; International 22q11.2 Deletion Syndrome Consortium: Practical guidelines for managing patients with $22 \mathrm{q} 11.2$ deletion syndrome. J Pediatr 2011;159:332-339.

9 Driscoll DA, Salvin J, Sellinger B, Budarf ML, McDonald-McGinn DM, Zackai EH, Emanuel BS: Prevalence of 22q11 microdeletions in DiGeorge and velocardiofacial syndromes: implications for genetic counselling and prenatal diagnosis. J Med Genet 1993;30:813817.

10 McDonald-McGinn DM, Tonnesen MK, Laufer-Cahana A, Finucane B, Driscoll DA, Emanuel BS, Zackai EH: Phenotype of the 22q11.2 deletion in individuals identified through an affected relative: cast a wide FISHing net. Genet Med 2001;3:23-29.

11 Grati FR, Molina Gomes D, Ferreira JC, Dupont C, Alesi V, Gouas L, Horelli-Kuitunen N, Choy KW, García-Herrero S, de la Vega AG, Piotrowski K, Genesio R, Queipo G,
Malvestiti B, Hervé B, Benzacken B, Novelli A, Vago P, Piippo K, Leung TY, Maggi F, Quibel T, Tabet AC, Simoni G, Vialard F: Prevalence of recurrent pathogenic microdeletions and microduplications in over 9,500 pregnancies. Prenat Diagn 2015;35: 801-809.

12 Wapner RJ, Martin CL, Levy B, Ballif BC, Eng CM, Zachary JM, Savage M, Platt LD, Saltzman D, Grobman WA, Klugman S, Scholl T, Simpson JL, McCall K, Aggarwal VS, Bunke B, Nahum O, Patel A, Lamb AN, Thom EA, Beaudet AL, Ledbetter DH, Shaffer LG, Jackson L: Chromosomal microarray versus karyotyping for prenatal diagnosis. N Engl J Med 2012;367:2175-2184.

13 Rauch A, Hoyer J, Guth S, Zweier C, Kraus C, Becker C, Zenker M, Hüffmeier U, Thiel C, Rüschendorf F, Nürnberg P, Reis A, Trautmann U: Diagnostic yield of various genetic approaches in patients with unexplained developmental delay or mental retardation. Am J Med Genet A 2006;140:2063-2074.

14 Sparks AB, Wang ET, Struble CA, Barrett W, Stokowski R, McBride C, Zahn J, Lee K, Shen N, Doshi J, Sun M, Garrison J, Sandler J, Hollemon D, Pattee P, Tomita-Mitchell A, Mitchell M, Stuelpnagel J, Song K, Oliphant A: Selective analysis of cell-free DNA in maternal blood for evaluation of fetal trisomy. Prenat Diagn 2012;32:3-9.

15 Juneau K, Bogard PE, Huang S, Mohseni M, Wang ET, Ryvkin P, Kingsley C, Struble CA, Oliphant A, Zahn JM: Microarray-based cellfree DNA analysis improves noninvasive prenatal testing. Fetal Diagn Ther 2014;36:282286.

16 Sparks AB, Struble CA, Wang ET, Song K, Oliphant A: Noninvasive prenatal detection and selective analysis of cell-free DNA obtained from maternal blood: evaluation for trisomy 21 and trisomy 18. Am J Obstet Gynecol 2012;206:319.e1-e9.

17 Barrett AN, McDonnell TC, Chan KC, Chitty LS: Digital PCR analysis of maternal plasma for noninvasive detection of sickle cell anemia. Clin Chem 2012;58:1026-1032.

18 Benn P, Cuckle H: Theoretical performance of non-invasive prenatal testing for chromosome imbalances using counting of cell-free
DNA fragments in maternal plasma. Prenat Diagn 2014;34:778-783.

19 Lo KK, Karampetsou E, Boustred C, McKay F, Mason S, Hill M, Plagnol V, Chitty LS: Limited clinical utility of non-invasive prenatal testing for subchromosomal abnormalities. Am J Hum Genet 2016;98:34-44.

20 Gross SJ, Ryan A, Benn P: Noninvasive prenatal testing for 22q11.2 deletion syndrome: deeper sequencing increases the positive predictive value. Am J Obstet Gynecol 2015;213: 254-255.

21 Wapner RJ, Babiarz JE, Levy B, Stosic M, Zimmermann B, Sigurjonsson S, Wayham N, Ryan A, Banjevic M, Lacroute P, Hu J, Hall MP, Demko Z, Siddiqui A, Rabinowitz M, Gross SJ, Hill M, Benn P: Expanding the scope of noninvasive prenatal testing: detection of fetal microdeletion syndromes. Am J Obstet Gynecol 2015;212:332.e1-e9.

22 Gross SJ, Stosic M, McDonald-McGinn DM, Bassett AS, Norvez A, Dhamankar R, Kobara K, Kirkizlar E, Zimmermann B, Wayham N, Babiarz JE, Ryan A, Jinnett KN, Demko Z, Benn P: Clinical experience with single-nucleotide polymorphism-based non-invasive prenatal screening for 22q11.2 deletion syndrome. Ultrasound Obstet Gynecol 2016;47: 177-183.

23 Helgeson J, Wardrop J, Boomer T, Almasri E, Paxton WB, Saldivar JS, Dharajiya N, Monroe TJ, Farkas DH, Grosu DS, McCullough RM: Clinical outcome of subchromosomal events detected by whole-genome noninvasive prenatal testing. Prenat Diagn 2015;35:9991004.

24 Shaikh TH, Kurahashi H, Saitta SC, O'Hare AM, Hu P, Roe BA, Driscoll DA, McDonaldMcGinn DM, Zackai EH, Budarf ML, Emanuel BS: Chromosome 22-specific low copy repeats and the 22q11.2 deletion syndrome: genomic organization and deletion endpoint analysis. Hum Mol Genet 2000;9:489-501.

25 Neofytou MC, Tsangaras K, Kypri E, Loizides C, Ioannides M, Achilleos A, Mina P, Keravnou A, Sismani C, Koumbaris G, Patsalis PC: Targeted capture enrichment assay for noninvasive prenatal testing of large and small size sub-chromosomal deletions and duplications. PLoS One 2017;12:e0171319. 